\title{
High-power short-duration versus standard-power standard-duration settings for repeat atrial fibrillation ablation
}

\author{
Joey Junarta ${ }^{1}$ Sean J. Dikdan ${ }^{1} \cdot$ Naman Upadhyay $^{1} \cdot$ Sairamya Bodempudi $^{1} \cdot$ Michael Y. Shvili $^{2} \cdot$ Daniel R. Frisch $^{1}$ (i)
}

Received: 11 September 2021 / Accepted: 5 November 2021 / Published online: 30 November 2021

(c) The Author(s) 2021

\begin{abstract}
Introduction High-power short-duration (HPSD) ablation is a novel strategy using contact force-sensing catheters optimized for radiofrequency ablation for atrial fibrillation (AF). No study has directly compared HPSD versus standard-power standard-duration (SPSD) contact force-sensing settings in patients presenting for repeat ablation with AF recurrence after initial ablation.

Methods We studied consecutive cases of patients with AF undergoing repeat ablation with SPSD or HPSD settings after their initial pulmonary vein isolation (PVI) with temperature controlled non-contact force, SPSD or HPSD settings between 6/23/14 and 3/4/20. Procedural data collected included radiofrequency ablation delivery time (RADT). Clinical data collected include sinus rhythm maintenance post-procedure.

Results A total of 61 patients underwent repeat ablation (36 SPSD, 25 HPSD). A total of 51 patients (83.6\%) were found to have pulmonary vein reconnections necessitating repeat isolation, 10 patients (16.4\%) had durable PVI and ablation targeted non-PV sources. RADT was shorter when comparing repeat ablation using HPSD compared to SPSD (22 vs 35 min; $p=0.01)$. There was no difference in sinus rhythm maintenance by Kaplan-Meier survival analysis (log rank test $p=0.87)$, after 3 or 12-months between groups overall, and when stratified by AF type, left atrial volume index, $\mathrm{CHA}_{2} \mathrm{DS}_{2}-\mathrm{VASc}$ score, or left ventricular ejection fraction.

Conclusion We demonstrated that repeat AF ablation with HPSD reduced procedure times with similar sinus rhythm maintenance compared to SPSD in those presenting for repeat ablation.
\end{abstract}

Keywords Catheter ablation $\cdot$ Atrial fibrillation $\cdot$ Recurrent atrial fibrillation $\cdot$ Electrophysiology

\section{Introduction}

The foundation of radiofrequency (RF) catheter ablation for atrial fibrillation lies in the electrical isolation of the pulmonary veins (PVI) from the left atrium [1,2]. This technique is proven to be effective and safe in reducing AF burden [1, 2]. Despite durable PVI after ablation, AF can recur. Repeat studies often demonstrate PV reconnections. However, at times, AF recurs even with durable PVI. The mechanism behind PV reconnection is incompletely understood, though

Daniel R. Frisch

daniel.frisch@jefferson.edu

1 Department of Medicine, Jefferson Heart Institute, Thomas Jefferson University Hospital, 925 Chestnut Street, Mezzanine level, Philadelphia, PA 19107, USA

2 Sidney Kimmel Medical College, Thomas Jefferson University, Philadelphia, USA incomplete ablation with partial thickness lesions or reversible injury is thought to be an important contributor [3-5].

Recently, there has been increasing interest in the use of contact force-guided (CF) high-power (45 to $70 \mathrm{~W}$ ) shortduration (5 to $15 \mathrm{~s}$ ) (HPSD) ablation for AF to reduce its recurrence and shorten ablation times. With standard-power ( 20 to $40 \mathrm{~W}$ ) standard-duration (20 to $60 \mathrm{~s}$ ) (SPSD) settings, the incidence of AF recurrence rate is about $15 \%$ at 3 months [6, 7]. Increasing the power to 45 to $50 \mathrm{~W}$ has been shown to improve ablation outcomes, but increasing power without shortening delivery duration has been associated with increased complications [8]. As such, other reports have used $50 \mathrm{~W}$ lesions for shorter durations, which led to improved ablation outcomes without increasing complications [9].

Three recent meta-analyses of observational studies comparing HPSD versus SPSD for initial AF ablation have yielded conflicting results [10-12]. There have been only 
two randomized controlled trials (RCT) comparing HPSD and SPSD settings thus far, which have also yielded inconsistent results with regards to sinus rhythm maintenance [13, 14]. Whether HPSD allows for improved freedom from AF after initial ablation is still unclear.

To our knowledge, there are no studies directly comparing HPSD versus SPSD settings in patients presenting for repeat ablation with AF recurrence after initial ablation. Previously, we compared clinical and procedural outcomes between temperature-controlled non-contact force (TCNC), SPSD, and HPSD settings at our institution $[15,16]$. In this report, we compared the long term clinical and procedural outcomes between HPSD and SPSD for repeat ablation in those with $\mathrm{AF}$ recurrence after initial AF ablation.

\section{Methods}

\section{Study population and design}

This consecutive case series included patients with paroxysmal or persistent AF referred for repeat ablation of AF between June 23, 2014 and March 4, 2020. Patients were eligible if they were undergoing their first repeat RF ablation with a strategy of SPSD or HPSD after undergoing an initial AF ablation with TCNC, SPSD, or HPSD settings. Patients were excluded if they underwent ablation for any other arrhythmia, if they presented for repeat ablation for AF beyond their first one, or if an ablation modality other than RF was used. Data on procedural and clinical characteristics were collected from our institution's electronic health record and stored in a secure password-protected database. The study was approved by our institutional review board.

\section{Catheter ablation procedure}

Written, informed consent was provided by all patients before the procedure in accordance with institutional policy. Antiarrhythmic drugs except amiodarone were stopped 3 days prior to the procedure. We have previously described our ablation protocol in separate studies $[15,16]$. Briefly, femoral venous access was obtained, then a multipolar catheter was placed in the coronary sinus. Afterwards, we place a diagnostic intra-cardiac ultrasound catheter $(5.5-10 \mathrm{MHz}$, AcuNav, Biosense Webster, Diamond Bar, CA or ViewFlex $^{\mathrm{TM}}$, Abbott Medical, St. Paul, MN) in the right atrium. Two inter-atrial trans-septal punctures were performed, then, an ablation catheter as well as a circular mapping catheter (Spiral, St. Jude Medical, St. Paul, MN) were advanced into the left atrium. Three-dimensional electroanatomic mapping was performed using the St. Jude EnSite ${ }^{\mathrm{TM}}$ Velocity $^{\mathrm{TM}}$ system (LSI, St. Jude Medical, St. Paul, MN), which is capable of recording the LSI during ablation.
Pulmonary veins were routinely isolated as a pair for initial ablation at our institution. Initial ablation was performed in the carina between ipsilateral veins if isolation could not be achieved with wide area encirclement. During repeat ablation, the pulmonary veins were first mapped to determine durability of electric isolation. Targeted PVI was conducted if reconnection occurred. In the case that pulmonary veins were durably isolated with initial mapping, the ablation strategy utilized depended on electroanatomic mapping and burst pacing inducibility for AF. Ablation targets included the anterior left atrial wall, posterior left atrial wall, left atrial roof, anterior mitral isthmus, posterior mitral isthmus, interatrial septum, cavo tricuspid isthmus, and/or coronary sinus. Generally, anterior ablation was performed if there was evidence of a re-entrant circuit or focal tachycardia originating from the anterior left atrial wall. For posterior wall ablation, either a circuit was identified on the posterior wall or high frequency, low amplitude signals were identified and targeted. Typically, the intention was complete posterior wall isolation when the posterior wall was targeted.

RF ablation for SPSD and HPSD was delivered with a 3.5-mm open-irrigated CF sensing catheter (TactiCath, St. Jude Medical, St. Paul, MN). Our SPSD protocol involved ablating with a flow of $17 \mathrm{cc} /$ minute for 30-60 s, with a power of 20-25 W, at a goal of 10-40 g per lesion, and a goal of 400-500 g seconds per site, with a LSI of 4.5-5.5. Our HPSD protocol involved administering RF ablation with a flow of $30 \mathrm{cc} /$ minute for up to $15 \mathrm{~s}$, with a power of $50 \mathrm{~W}$, at a goal of 8-40 g per lesion, guided by a LSI of 6 on the anterior left atrium and an LSI of 5 on the posterior left atrium. In all cases, esophageal temperature monitoring was arranged and lesions were aborted if the temperature rose by $0.2{ }^{\circ} \mathrm{C}$ or more.

Successful PVI was defined by the loss of all PV potentials (entrance block) and failure to capture the left atrium when pacing from sequential bipoles of the circular mapping catheter placed at the ostium of each PV (exit block; 10 millivolts were delivered with a $2 \mathrm{~ms}$ pulse width with each pacing stimulus). Attempts at reinduction with burst pacing were performed.

\section{Follow up}

Patients were routinely followed-up at 1, 3, 6, and 12 months after their repeat ablation to assess for clinical outcomes. To determine AF status, patients' reports of symptoms and electrocardiography were evaluated at each follow-up visit. We ensured conducting a high quality 12-lead electrocardiographic reading to inform further management of the patient's AF at each visit. Mobile cardiac outpatient telemetry monitors were utilized if patients had signs or symptoms concerning for recurrence of their AF, including if they were intermittently 
symptomatic with chest pain, shortness of breath, palpitations, near syncope, or dizziness. Patients were also encouraged to report symptoms via telephone, email, or electronic medical record messaging.

\section{Study endpoints}

Primary procedural endpoints include RF ablation delivery time (RADT) and the inducibility of arrhythmias after ablation. RADT is the total time that RF ablation was delivered and not the time in between lesions. Primary clinical endpoints included the recurrence of AF in the first 3 months and 12 months after ablation as well as the probability of AF recurrence over 12 months by Kaplan-Meier survival analysis. Recurrence of AF was defined as $\geq 30 \mathrm{~s}$ of asymptomatic or symptomatic AF.

\section{Statistical analyses}

Means of continuous variables were analyzed with the student's $t$ test. The non-parametric Wilcoxon-Mann Whitney test was used to compare the median of variables. Categorical variables were analyzed using a Chisquared test. The paired $t$ test was used to compare the use of medications within the same group over the follow-up period. Kaplan-Meier curves and the log-rank test was used to compare atrial fibrillation recurrence. A twosided $p$ value of $<0.05$ was used to determine statistical significance. Analyses were performed using STATA/SE 16.1 (College Station, TX, USA).

\section{Results}

\section{Baseline characteristics}

Clinical and baseline characteristics of included patients are shown in Table 1. There was no difference in age, sex, type of $\mathrm{AF}, \mathrm{CHA}_{2} \mathrm{DS}_{2}$-VASc score, anti-arrhythmic drug use at different follow-up time periods, left atrial volume index (LAVI), or left ventricular ejection fraction (LVEF) between groups. There was a difference in anticoagulation use at study recruitment between HPSD and SPSD groups $(100.0 \%$ vs. $80.0 \% ; p=0.01)$. However, there was no difference in anticoagulation use at $3(p=0.07)$ and 12-month $(p=0.18)$ follow-up.

\section{Procedural outcomes}

A total of 24 patients (39.3\%) underwent their initial RF ablation with TCNC. A total of 34 (55.7\%) and 3 (4.9\%) patients underwent initial RF ablation with SPSD and HPSD settings, respectively. Upon evaluation during repeat ablation, 51 patients $(83.6 \%)$ were found to have pulmonary vein reconnections necessitating repeat isolation, while 10 patients (16.4\%) had durable PVI and ablation targeted nonPV sources (Supplementary Table 1). Non-PV sources that were targeted included ablation of the anterior and posterior mitral isthmus, interatrial septum, coronary sinus, left atrial roof line, anterior left atrial wall, posterior left atrial wall, and cavo tricuspid isthmus.

Table 2 and Fig. 1 compares procedural outcomes between catheter settings. There was a difference in RADT between groups $(p=0.01)$. HPSD settings reduced ablation

Table 1 Clinical characteristics

\begin{tabular}{|c|c|c|c|}
\hline & $\begin{array}{l}\text { Standard-power standard-dura- } \\
\text { tion }(N=36)\end{array}$ & $\begin{array}{l}\text { High-power short-duration } \\
(N=25)\end{array}$ & $p$ value \\
\hline Age in years, mean $(\mathrm{SD})$ & $61.0(1.7)$ & $63.4(1.7)$ & 0.35 \\
\hline Male sex, no. (\%) & $22(61.1 \%)$ & $13(52.0 \%)$ & 0.48 \\
\hline Paroxysmal atrial fibrillation, no. (\%) & $13(36.1 \%)$ & $14(56.0 \%)$ & 0.16 \\
\hline $\mathrm{CHA}_{2} \mathrm{DS}_{2}$-VASc score, median (IQR) & $2(1-3)$ & $1.5(1-3)$ & 0.85 \\
\hline Antiarrhythmic drug use, no. (\%) & $31(86.1 \%)$ & $20(80.0 \%)$ & 0.53 \\
\hline Antiarrhythmic drug use at 3 months follow-up, no. (\%) & $27(75.0 \%)$ & $20(80.0 \%)$ & 0.44 \\
\hline Antiarrhythmic drug use at 12 months follow-up, no. (\%) & $22(61.1 \%)$ & $17(68.0 \%)$ & 0.38 \\
\hline Anticoagulant use, no. (\%) & $36(100.0 \%)$ & $20(80.0 \%)$ & 0.01 \\
\hline Anticoagulant use at 3 months follow-up, no. (\%) & $31(86.1 \%)$ & $16(64.0 \%)$ & 0.07 \\
\hline Anticoagulant use at 12 months follow-up, no. (\%) & $27(75.0 \%)$ & $14(56.0 \%)$ & 0.18 \\
\hline Left atrial volume index, mean (SD) & $36.9(2.4)$ & $35.7(2.6)$ & 0.76 \\
\hline Left ventricular ejection fraction $\%$, mean (SD) & $58.8(12.7)$ & $61.0(12.2)$ & 0.51 \\
\hline
\end{tabular}

$N$ number of participants, $S D$ standard deviation, no. number, $I Q R$ inter-quartile range 
Table 2 Procedural time by catheter type

\begin{tabular}{lll}
\hline & \multicolumn{2}{l}{ Radiofrequency ablation delivery time } \\
\cline { 2 - 3 } & $\begin{array}{l}\text { Standard-power standard- } \\
\text { duration }\end{array}$ & $\begin{array}{l}\text { High-power } \\
\text { short-dura- } \\
\text { tion }\end{array}$ \\
\hline $\mathrm{N}$ & 36 & 25 \\
Mean & $00: 35: 03$ & $00: 21: 38$ \\
SD & $00: 21: 56$ & $00: 15: 12$ \\
p value & 0.01 & \\
\hline
\end{tabular}

$N$ number of participants, $S D$ standard deviation

times by about $13 \mathrm{~min}$. There was no difference in the ability to reinduce arrhythmias between catheter types (Table 3).

\section{Clinical outcomes}

AF recurrence was assessed at 3 months and 12 months after ablation. There was no difference in the overall percentage of patients in sinus rhythm between groups at 3 months (Table 4) or 12 months (Table 5). Notably, the number of patients in both groups decreased over time as they were lost to follow-up. There was no difference in AF recurrence when patients were stratified by AF type, LAVI, $\mathrm{CHA}_{2} \mathrm{DS}_{2}$-VASc score, or LVEF at 3 months or 12 months. Time to first AF recurrence for each patient was assessed and showed no difference between groups over 12 months by Kaplan-Meier survival analysis (log rank test $p=0.87$ ) shown in Fig. 2.
Table 3 Arrhythmia inducibility by ablation strategy

\begin{tabular}{llll}
\hline & $\begin{array}{l}\text { Standard-power } \\
\text { standard-duration } \\
(N=36)\end{array}$ & $\begin{array}{l}\text { High-power } \\
\text { short-duration } \\
(N=25)\end{array}$ & $p$ value \\
\hline Non-inducible & 20 & 14 & 0.32 \\
$\begin{array}{l}\text { Atrial fibrillation } \\
\begin{array}{c}\text { Other supraven- } \\
\text { tricular } \\
\text { arrhythmia }\end{array}\end{array}$ & 7 & 9 & \\
\hline
\end{tabular}

$N$ number of participants

Table 4 Patients in sinus rhythm after 3 months based on clinical characteristics and catheter type

\begin{tabular}{llll}
\hline & SPSD $(N=35)$ & HPSD $(N=24)$ & $p$ value \\
\hline $\begin{array}{l}\text { Overall patients in } \\
\text { sinus rhythm, no. }\end{array}$ & $27(77.1 \%)$ & $19(79.2 \%)$ & 0.85 \\
$(\%)$ & & \\
Type of atrial fibrillation, no & & \\
Paroxysmal & 10 & 11 & 0.76 \\
Persistent & 18 & 9 & \\
Left atrial volume index, no & & \\
$\geq 35$ & 10 & 7 & \\
$<34$ & 10 & 7 & \\
$\mathrm{CHA}_{2} \mathrm{DS}_{2}$-VASc score, no & & \\
$\geq 2$ & 17 & 12 & \\
$<2$ & 10 & 8 & \\
Left ventricular ejection fraction $\%$, no & & \\
$\geq 55$ & 17 & 17 & \\
$<55$ & 9 & 3 & \\
\hline
\end{tabular}

SPSD standard-power standard-duration, HPSD high-power shortduration, $N$ number of participants, $n o$. number
Fig. 1 Radiofrequency ablation delivery time by ablation settings. SPSD standard-power standard-duration, HPSD highpower short-duration
$00: 36: 00$

$00: 28: 48$

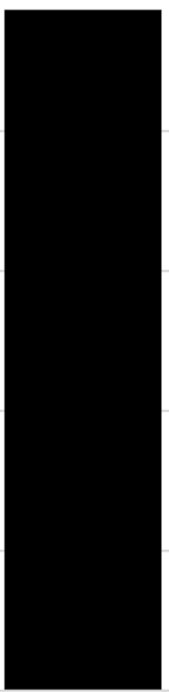

SPSD

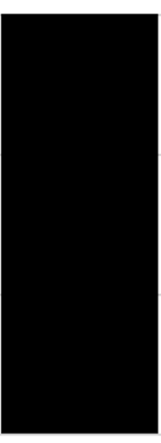

HPSD 
Table 5 Patients in sinus rhythm after 12 months based on clinical characteristics and catheter type

\begin{tabular}{|c|c|c|c|}
\hline & $\operatorname{SPSD}(N=33)$ & $\operatorname{HPSD}(N=24)$ & $p$ value \\
\hline $\begin{array}{l}\text { Overall patients in } \\
\text { sinus rhythm, no. } \\
(\%)\end{array}$ & $23(69.7 \%)$ & $15(62.5 \%)$ & 0.57 \\
\hline \multicolumn{4}{|c|}{ Type of atrial fibrillation, no } \\
\hline Paroxysmal & 10 & 10 & 0.64 \\
\hline Persistent & 13 & 5 & \\
\hline \multicolumn{4}{|c|}{ Left atrial volume index, no } \\
\hline$\geq 35$ & 11 & 4 & 0.26 \\
\hline$<34$ & 8 & 7 & \\
\hline \multicolumn{4}{|c|}{$\mathrm{CHA}_{2} \mathrm{DS}_{2}$-VASc score, no } \\
\hline$\geq 2$ & 17 & 9 & 0.45 \\
\hline$<2$ & 6 & 6 & \\
\hline \multicolumn{4}{|c|}{ Left ventricular ejection fraction $\%$, no } \\
\hline$\geq 55$ & 17 & 13 & 0.46 \\
\hline$<55$ & 6 & 2 & \\
\hline
\end{tabular}

SPSD standard-power standard-duration, HPSD high-power shortduration, $N$ number of participants, no. number

\section{Adverse events}

No adverse events occurred. No pericardial effusion, esophageal injuries, phrenic nerve injuries, bleeding requiring transfusion, strokes, or deaths occurred in any groups.

\section{Discussion}

Our study demonstrates that HPSD reduced procedural times compared to SPSD ablation with no difference in sinus rhythm maintenance in patients presenting for repeat $\mathrm{RF}$ ablation for AF. Additionally, there was no difference in sinus rhythm maintenance when stratified by AF type, left atrial volume index, $\mathrm{CHA}_{2} \mathrm{DS}_{2}$-VASc score, or left ventricular ejection fraction.

There are two heating phases that constitute the basis of $\mathrm{RF}$ ablation lesion formation: resistive and conductive heating. The electrical current delivered during resistive heating leads to immediate and irreversible injury to superficial tissue layers at temperatures of $>50{ }^{\circ} \mathrm{C}$. During the resistive heating phase, a heat source created passively extends into deeper tissue layers at lower temperatures of $<50{ }^{\circ} \mathrm{C}$, this is known as conductive heating. It has been shown that highpower RF ( 45 to $70 \mathrm{~W}$ ) delivery with shorter duration ( 5 to $15 \mathrm{~s})$ may lead to a shift allowing increased resistive heating and decreased conductive heating, and be sufficiently adequate in creating effective ablation lesions [17, 18]. The superiority and safety of HPSD is of considerable interest.

Three meta-analyses of observational studies and two RCTs comparing HPSD versus SPSD for initial AF ablation have yielded conflicting results [10-14]. The meta-analyses included anywhere between 10 and 15 studies involving 2274 to 3718 patients [10-12]. Two showed a difference in freedom from atrial arrhythmia with HPSD when compared to conventional RF settings $[10,11]$. However, the metaanalysis by Kewcharoen et al. did not [12]. Nevertheless,
Fig. 2 Kaplan-Meier survival analysis for atrial fibrillation recurrence. SPSD standardpower standard-duration, HPSD high-power short-duration

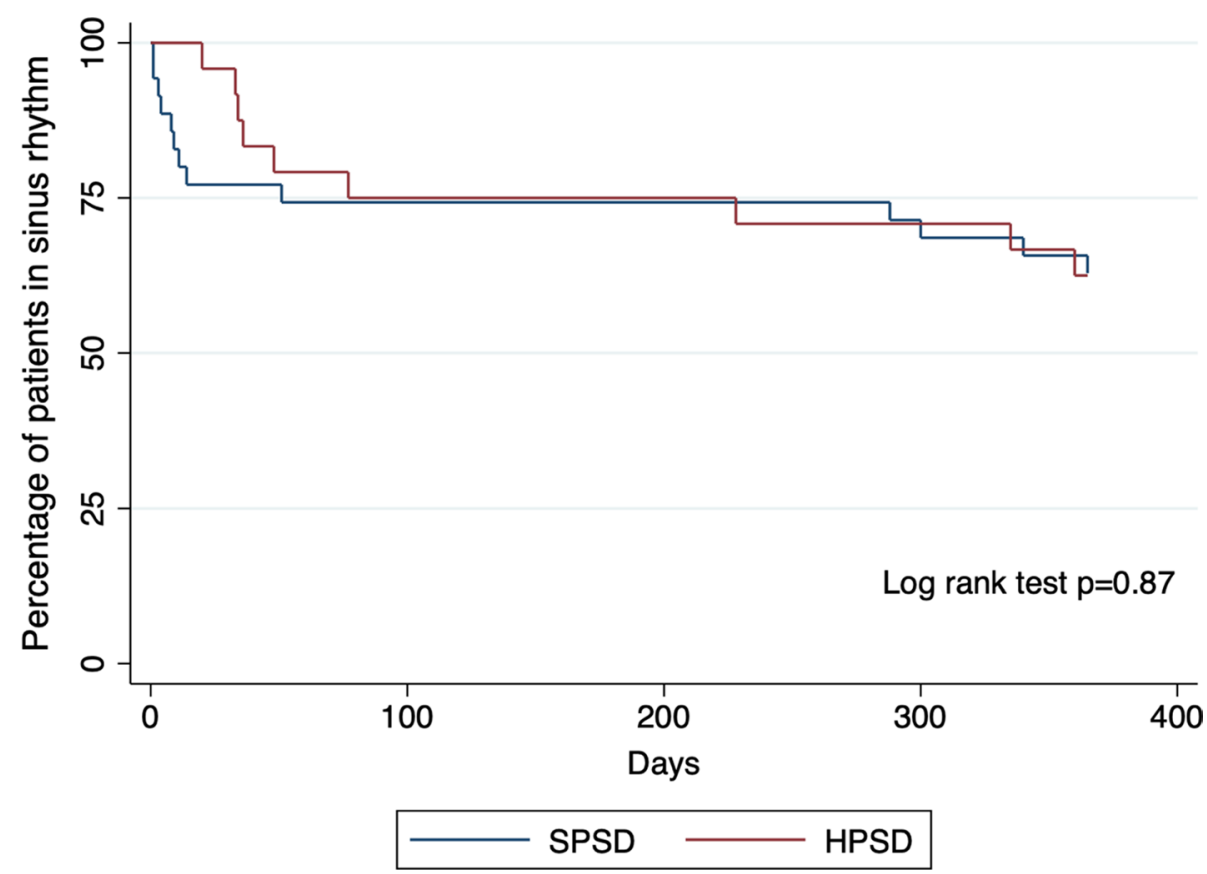


these studies were consistent in demonstrating reduced RF ablation time with HPSD settings and showed no difference in peri-procedural complications.

Less studied are the optimal ablation parameters that should be used for ablation of recurrent AF. In one single cohort study, the outcome of repeat RF ablation with $35 \mathrm{~W}$ of energy after initial cryoballoon ablation was evaluated [19]. By means of Kaplan-Meier analysis, freedom from AF was $94 \%$ and $86 \%$ at 12 months and 24 months after redo ablation, respectively. Pokushalov et al. compared cryoballoon versus RF ablation of paroxysmal AF after failed initial $\mathrm{RF}$ ablation [20]. Repeat AF ablation was done with $35 \mathrm{~W}$ of energy. By intention-to-treat analysis, $58 \%$ of the RF group vs $43 \%$ of the cryoballoon group ( $p=0.06$ ) patients were AF-free on no antiarrhythmics. With regards to on-treatment comparisons, $53 \%$ of patients in the RF group and $38 \%$ of the cryoballoon group $(p=0.04)$ were AF-free at 12-month follow-up. This study suggests that RF may be preferable to cryoballoon as a redo ablation strategy.

No studies have directly compared HPSD versus SPSD settings in patients presenting for repeat ablation with $\mathrm{AF}$ recurrence after initial RF ablation. We demonstrate that HPSD shortened ablation times without sacrificing efficacy. Perhaps not surprisingly, our study did not show improved clinical outcomes using HPSD in patients presenting for redo ablation. It is important to emphasize that although suggestive, there is no convincing randomized evidence that HPSD ablation allows for improved long-term outcomes, be it for initial or redo ablations.

A further observation is the recurrence pattern based on the strategy used for the initial ablation. Despite the development of CF measurements, automated lesion assessment, and next-generation catheters, AF recurrence after PVI remains a significant challenge. Here, we showed that most patients presenting for redo ablation had evidence of PV reconnection (83.6\%). Herein lies the interest in optimizing RF settings for improved outcomes after RF ablation.

\section{Limitations}

Our study had a small sample size, which meant that it was difficult to detect a significant difference in clinical outcomes between groups. Next, it is possible that some patients had undetected AF recurrence, which could falsely elevate the rate of sinus rhythm maintenance in the study. This is despite close follow-up, outpatient electrocardiographic monitoring, and telemetry monitoring, as rhythm monitoring was not continuous. There was a baseline difference in anticoagulant use between groups. Importantly, there was no difference in the use of anticoagulant drugs at 3 and 12 months between groups, which minimizes any potential impact this may have on confounding the long-term maintenance of sinus rhythm attributed to the different ablation strategies.

\section{Conclusion}

To the best of our knowledge, this is the first study to compare HPSD versus SPSD settings in patients presenting for repeat $\mathrm{AF}$ ablation after $\mathrm{AF}$ recurrence. We found that $\mathrm{AF}$ ablation with HPSD settings guided by LSI reduced procedure times with similar sinus rhythm maintenance and safety profile compared to SPSD for those undergoing their first redo ablation. Further large RCTs will help clarify whether HPSD settings improve not only procedural times, but long term sinus rhythm maintenance in those presenting for initial and redo RF ablation for $\mathrm{AF}$.

Supplementary Information The online version contains supplementary material available at https://doi.org/10.1007/s00380-021-01987-9.

Author contributions DF design, data collection, manuscript, supervision. JJ data collection, analysis, manuscript. SD data collection, analysis, manuscript. NU data collection, analysis, manuscript. SB data collection, analysis, manuscript. MS data collection, analysis, manuscript.

Funding The authors have no sources of funding for this research to declare.

Availability of data and materials Data are safely kept in a password protected security system at Thomas Jefferson University Hospital. The datasets used and/or analysed during the current study are de-identified and available from the corresponding author on reasonable request.

Code availability Not applicable.

\section{Declarations}

Conflict of interest The authors declare that they have no competing interests. The results presented in this paper have not been published previously in whole or part, except in abstract form.

Ethical approval and consent to participate All procedures performed in studies involving human participants were in accordance with the ethical standards of the institutional and/or national research committee and with the 1964 Helsinki declaration and its later amendments or comparable ethical standards. The study was approved by our institutional review board. This article does not contain any studies with animals performed by any of the authors.

Consent for publication Not applicable.

Open Access This article is licensed under a Creative Commons Attribution 4.0 International License, which permits use, sharing, adaptation, distribution and reproduction in any medium or format, as long as you give appropriate credit to the original author(s) and the source, provide a link to the Creative Commons licence, and indicate if changes were made. The images or other third party material in this article are included in the article's Creative Commons licence, unless indicated otherwise in a credit line to the material. If material is not included in the article's Creative Commons licence and your intended use is not permitted by statutory regulation or exceeds the permitted use, you will need to obtain permission directly from the copyright holder. To view a copy of this licence, visit http://creativecommons.org/licenses/by/4.0/. 


\section{References}

1. Kirchhof P, Benussi S, Kotecha D, Ahlsson A, Atar D, Casadei B, Castella M, Diener HC, Heidbuchel H, Hendriks J, Hindricks G, Manolis AS, Oldgren J, Popescu BA, Schotten U, Van Putte B, Vardas P, ESC Scientific Document Group (2016) 2016 ESC guidelines for the management of atrial fibrillation developed in collaboration with EACTS. Eur Heart J 37:2893-2962

2. Calkins H, Hindricks G, Cappato R, Kim YH, Saad EB, Aguinaga L, Akar JG, Badhwar V, Brugada J, Camm J, Chen PS, Chen SA, Chung MK, Nielsen JC, Curtis AB, Davies DW, Day JD, d'Avila A, de Groot NMSN, Di Biase L, Duytschaever M, Edgerton JR, Ellenbogen KA, Ellinor PT, Ernst S, Fenelon G, Gerstenfeld EP, Haines DE, Haissaguerre M, Helm RH, Hylek E, Jackman WM, Jalife J, Kalman JM, Kautzner J, Kottkamp H, Kuck KH, Kumagai K, Lee R, Lewalter T, Lindsay BD, Macle L, Mansour M, Marchlinski FE, Michaud GF, Nakagawa H, Natale A, Nattel S, Okumura K, Packer D, Pokushalov E, Reynolds MR, Sanders P, Scanavacca M, Schilling R, Tondo C, Tsao HM, Verma A, Wilber DJ, Yamane T (2018) 2017 HRS/EHRA/ECAS/APHRS/ SOLAECE expert consensus statement on catheter and surgical ablation of atrial fibrillation: executive summary. Europace 20:157-208

3. Andrade JG, Monir G, Pollak SJ, Khairy P, Dubuc M, Roy D, Talajic M, Deyell M, Rivard L, Thibault B, Guerra PG, Nattel S, Macle L (2014) Pulmonary vein isolation using "contact force" ablation: the effect on dormant conduction and long-term freedom from recurrent atrial fibrillation-a prospective study. Heart Rhythm 11:1919-1924

4. Anter E, Contreras-Valdes FM, Shvilkin A, Tschabrunn CM, Josephson ME (2014) Acute pulmonary vein reconnection is a predictor of atrial fibrillation recurrence following pulmonary vein isolation. J Interv Card Electrophysiol 39:225-232

5. Anter E, Tschabrunn CM, Contreras-Valdes FM, Buxton AE, Josephson ME (2014) Radiofrequency ablation annotation algorithm reduces the incidence of linear gaps and reconnection after pulmonary vein isolation. Heart Rhythm 11:783-790

6. Ullah W, McLean A, Tayebjee MH, Gupta D, Ginks MR, Haywood GA, O’Neill M, Lambiase PD, Earley MJ, Schilling RJ, UK Multicentre Trials Group (2016) Randomized trial comparing pulmonary vein isolation using the SmartTouch catheter with or without real-time contact force data. Heart Rhythm 13:1761-1767

7. Kautzner J, Neuzil P, Lambert H, Peichl P, Petru J, Cihak R, Skoda J, Wichterle D, Wissner E, Yulzari A, Kuck KH (2015) EFFICAS II: optimization of catheter contact force improves outcome of pulmonary vein isolation for paroxysmal atrial fibrillation. Europace 17:1229-1235

8. Kanj MH, Wazni O, Fahmy T, Thal S, Patel D, Elayi C, Di Biase L, Arruda M, Saliba W, Schweikert RA, Cummings JE, Burkhardt JD, Martin DO, Pelargonio G, Dello Russo A, Casella M, Santarelli P, Potenza D, Fanelli R, Massaro R, Forleo G, Natale A (2007) Pulmonary vein antral isolation using an open irrigation ablation catheter for the treatment of atrial fibrillation: a randomized pilot study. J Am Coll Cardiol 49:1634-1641

9. Winkle RA, Mead RH, Engel G, Patrawala RA (2011) Atrial fibrillation ablation: "perpetual motion" of open irrigated tip catheters at $50 \mathrm{~W}$ is safe and improves outcomes. Pacing Clin Electrophysiol 34:531-539

10. Ravi V, Poudyal A, Abid Q, Larsen T, Krishnan K, Sharma PS, Trohman RG, Huang HD (2021) High-power short duration vs. conventional radiofrequency ablation of atrial fibrillation: a systematic review and meta-analysis. Europace 23(5):710-721

11. Chen CF, Wu J, Jin CL, Liu MJ, Xu YZ (2020) Comparison of high-power short-duration and low-power long-duration radiofrequency ablation for treating atrial fibrillation: systematic review and meta-analysis. Clin Cardiol 43(12):1631-1640

12. Kewcharoen J, Techorueangwiwat C, Kanitsoraphan C, Leesutipornchai T, Akoum N, Bunch TJ, Navaravong L (2021) Highpower short duration and low-power long duration in atrial fibrillation ablation: a meta-analysis. J Cardiovasc Electrophysiol 32(1):71-82

13. Shin DG, Ahn J, Han SJ, Lim HE (2020) Efficacy of high-power and short-duration ablation in patients with atrial fibrillation: a prospective randomized controlled trial. Europace 10:1495-1501

14. Leo M, Pedersen M, Rajappan K, Ginks MR, Hunter RJ, Bowers R, Kalla M, Bashir Y, Betts TR (2020) Power, lesion size index and oesophageal temperature alerts during atrial fibrillation ablation: a randomized study. Circ Arrhythm Electrophysiol 13(10): e008316

15. Frisch DR, Dikdan SJ (2019) Clinical and procedural effects of transitioning to contact force guided ablation for atrial fibrillation. J Atr Fibrillation 11:2081

16. Dikdan SJ, Junarta J, Bodempudi S, Upadhyay N, Pang Z, Frisch DR (2021) Comparison of clinical and procedural outcomes between high-power short-duration, standard-power standardduration, and temperature-controlled noncontact force guided ablation for atrial fibrillation. J Cardiovasc Electrophysiol 32(3):608-615

17. Leshem E, Zilberman I, Tschabrunn CM, Barkagan M, ContrerasValdes FM, Govari A, Anter E (2018) High-power and shortduration ablation for pulmonary vein isolation: biophysical characterization. JACC Clin Electrophysiol 4:467-479

18. Barkagan M, Contreras-Valdes FM, Leshem E, Buxton AE, Nakagawa H, Anter E (2018) High-power and short-duration ablation for pulmonary vein isolation: safety, efficacy, and long-term durability. J Cardiovasc Electrophysiol 29:1287-1296

19. Conte G, Chierchia GB, Sieira J, Levinstein M, Casado-Arroyo R, De Asmundis C, Sarkozy A, Rodriguez-Manero M, Di Giovanni G, Baltogiannis G, Wauters K, Brugada P (2013) Repeat procedure using radiofrequency energy for recurrence of atrial fibrillation after initial cryoballoon ablation: a 2-year follow-up. Europace 15(10):1421-1425

20. Pokushalov E, Romanov A, Artyomenko S, Baranova V, Losik D, Bairamova S, Karaskov A, Mittal S, Steinberg JS (2013) Cryoballoon versus radiofrequency for pulmonary vein re-isolation after a failed initial ablation procedure in patients with paroxysmal atrial fibrillation. J Cardiovasc Electrophysiol 24(3):274-279

Publisher's Note Springer Nature remains neutral with regard to jurisdictional claims in published maps and institutional affiliations. 